\section{Effects of social protection on tuberculosis treatment outcomes in low or middle-income and in high-burden countries: systematic review and meta-analysis}

\section{Efeitos da proteção social sobre os desfechos do tratamento da tuberculose em países de renda baixa e média ou de carga alta da doença: uma revisão sistemática e meta-análise}

\section{Efectos de la protección social en los resultados del tratamiento contra la tuberculosis en países con baja o media renta y gravemente afectados: revisión sistemática y metaanálisis}

\begin{abstract}
Tuberculosis $(T B)$ is a poverty infectious disease that affects millions of people worldwide. Evidences suggest that social protection strategies (SPS) can improve TB treatment outcomes. This study aimed to synthesize such evidences through systematic literature review and meta-analysis. We searched for studies conducted in low-or middle-income and in high TB-burden countries, published during 1995-2016. The review was performed by searching PubMed/MEDLINE, Scopus, Web of Science, ScienceDirect and LILACS. We included only studies that investigated the effects of SPS on TB treatment outcomes. We retained 25 studies for qualitative synthesis. Meta-analyses were performed with 9 randomized controlled trials, including a total of 1,687 participants. Pooled results showed that SPS was associated with TB treatment success $(R R=1.09$; 95\%CI: $1.03-1.14)$, cure of TB patients $(R R=1.11$; 95\%CI: 1.01-1.22) and with reduction in risk of TB treatment default $(R R=$ 0.63; 95\%CI: 0.45-0.89). We did not detect effects of SPS on the outcomes treatment failure and death. These findings revealed that SPS might improve $T B$ treatment outcomes in lower-middle-income economies or countries with high burden of this disease. However, the overall quality of evidences regarding these effect estimates is low and further well-conducted randomized studies are needed.
\end{abstract}

Tuberculosis; Treatment Outcome; Social Welfare; Public Policy
Kaio Vinicius Freitas de Andrade 1

Joilda Silva Nery 2

Ramon Andrade de Souza 1

Susan Martins Pereira 1

doi: 10.1590/0102-311X00153116
Correspondence

K. V.F. Andrade

Instituto de Saúde Coletiva, Universidade Federal da Bahia. Rua Basílio da Gama s/n, Salvador, BA 40110-040, Brasil. kaiovinnicius@yahoo.com.br

1 Instituto de Saúde Coletiva, Universidade Federal da Bahia, Salvador, Brasil.

2 Universidade Federal do Vale do São Francisco, Paulo Afonso, Brasil. 


\section{Introduction}

Tuberculosis (TB) is still considered a major global health problem, mainly in socially vulnerable population groups living in low- and middle-income countries 1. In 2015, 10.4 million new cases and 1.4 million deaths because of TB were estimated worldwide ${ }^{2}$. According to the United Nations Sustainable Development Goals (2016-2030), the World Health Organization (WHO) set a 2030 target of 90\% reduction in deaths, $80 \%$ decline in TB incidence and zero TB-affected families facing catastrophic costs because of this disease $3,4,5$.

Poverty is one of the most important determinants of TB and accounts for almost one third of the global burden of diseases. Besides being a poverty related illness, TB also worsens this social condition. In low-income countries, approximately $17 \%$ of TB-deaths affect the economically productive age group of 15-49 years 6 . In those countries, investments in prevention, diagnostics and treatment should consider social protection and urban planning interventions in order to improve access to TB care and treatment adherence 7,8 .

Social protection strategies (SPS) enable individuals and households to protect and build their capital assets, leading them to move structurally out of poverty 7,8,9. Their main components are social and income security, cash transfer programs, food provision, transport incentives, unemployment insurance, education, microcredit and income generation policies, as well as psychosocial support, protection against stigma/discrimination and public health systems with universal coverage, which are also included in the field of social protection 1,10,11.

Universal health coverage and social protection account for reduction of income losses and costs incurred by TB patients, leading to positive impacts on the reduction of this disease burden, measured by incidence, prevalence and mortality rates 1,2 . For these reasons, bold policies and supportive systems are among the most important pillars of the post-2015 global tuberculosis strategy 3,5.

In this context, the aim of this systematic review was to identify and assess evidences of social protection effects on TB treatment outcomes in low- and middle-income countries or in high TBburden countries.

\section{Methods}

Following the reporting guidelines recommended in PRISMA statement 12, we conducted a systematic review to identify studies that have investigated the effects of SPS on TB treatment outcomes. The review protocol was registered in PROSPERO international database on September 17, 2015 (registration number CRD42015026305).

\section{Eligibility criteria}

Eligibility of studies followed predetermined inclusion criteria. We included interventional (randomized controlled trials - RCTs and studies with quasi-experimental design) and observational studies covering the period from 1995 to May 31st, 2016. All included studies were developed in low- and middle-income countries, according to World Bank income classification ${ }^{13}$ or in one of the 30 high TB-burden countries listed by WHO 2.

We defined individuals under TB treatment as population of reviewed studies. The interventions of interest were SPS. Controls should be individuals under usual TB care. Primary outcome was TB treatment success rate (TSR), expressed by the proportion of all new cases that successfully completed treatment, with or without bacteriological evidences of cure 14 .

Secondary outcomes were: (a) cure rate, i.e. proportion of TB cases with a negative sputum smear result recorded during the last month of treatment and in at least one previous occasion during treatment; (b) default rate, i.e. proportion of TB cases that interrupted treatment for two consecutive months or more; (c) treatment failure rate, i.e. proportion of TB cases with smear positive results five months or later after initiating treatment; (d) death rate, i.e. the proportion of TB cases that died during treatment, irrespective of cause. All these outcomes should meet WHO international definitions 14 


\section{Search strategy}

We searched the electronic databases PubMed/MEDLINE, Scopus, Web of Science, ScienceDirect and LILACS, without language restriction. The search terms were defined according to Medical Subject Headings (MeSH) and Descriptors in Health Sciences (DeCS). Search strategy applied the terms: social protection, public policy, social welfare, income, food assistance, food supply, transportation, reimbursement, financial support, government financing, social work, social security, public assistance, motivation. We used the operator "OR" to connect synonyms and the operator "AND" to combine them with the term tuberculosis. We applied filters to humans and publication dates. The searches occurred in October 2015 and were rerun in May 2016.

We hand searched in clinical trials registries (Clinical Trials.gov and Brazilian Clinical Trials Registry - ReBEC). Google Scholar was also screened in order to access grey literature (e.g. non-indexed journals, official documents, government reports), abstracts published in annals of scientific meetings, theses and dissertations digital libraries. The full search strategy is available in the supplemental material - cf. Appendix 1: https://www.4shared.com/web/preview/pdf/B-Gv6byoca.

One reviewer (K.V.F.A.) conducted the literature search based on the strategy developed by all the review team. Then, two reviewers (K.V.F.A., R.A.S.) independently examined titles and abstracts. Cohen's Kappa coefficient (k) was used as a measure of inter-rater agreement. Disagreements were resolved by consensus or by consulting a third reviewer (S.M.P.) to adjudicate.

We extracted and summarized data from the fully reviewed studies using a form to list study characteristics, including: author, publication year, study design and location, criteria for inclusion and exclusion of participants, randomization approach (if applicable), description of interventions, and TB treatment outcomes.

\section{Quality assessment}

We adapted Downs \& Black 15 checklist to assess the methodological quality of non-randomized studies (NRS). The original version of this tool contains 27 items that assess internal (bias and confounding) and external validity (sample representativeness). Two reviewers (K.V.F.A., J.S.N.) performed the quality assessment applying 21 "yes"-or-"no" questions (using the scores 1 for yes and 0 for no) and another one with three answer options in order to check the description of confounding in each study, with these answer options: described ( 2 points), partially described (1 point) or not described ( 0 points). The total maximum score was 23 .

The reviewers excluded five questions of the original version 15 for considering them inadequate or not applicable for our quality assessment. These questions addressed the following features: reporting of random variability estimates in the data for the main outcome, reporting of adverse events associated with study interventions, presence of unplanned analyses, adequacy of statistical tests and study power. Some of these excluded questions have limitations in their answer options. The scores profile provided a summary of methodological strengths and weakness of each study. However, we did not exclude studies based on it.

We performed a specific quality assessment for RCTs using The Cochrane Risk of Bias Tool 16. In addition, quality of evidence across studies was evaluated with the Grading of Recommendations, Assessment, Development and Evaluation (GRADE) 17 approach. GRADEpro Guideline Development Tool (GDT) 18 was used to visualize evidences profile and generate the summary of findings (SoF) table.

\section{Data analysis}

All studies retained for systematic review were described in qualitative synthesis. Only RCTs were submitted to meta-analysis. TB treatment outcomes were analyzed using risk ratios (RR) and their $95 \%$ confidence intervals $(95 \% \mathrm{CI})$, recalculated from the data provided by RCTs, using the Mantel-Haenszel method. Meta-analyses were performed according to each outcome. Data from cluster-RCTs were adjusted based on the intracluster (or intraclass) correlation coefficient (ICC) provided by these studies 16 . 
To conduct these meta-analyses, a random effects model was fitted. This choice was based on the diversity of interventions and studies characteristics (e.g. participants and methods). The I2 statistic was used to quantify the heterogeneity, categorized as not important (I2 value between 0-40\%), moderate (I2 value between 30-60\%), substantial (I2 value between $50-90 \%$ ) and considerable (I 2 value of $75 \%$ or more) 16 . Visual inspection of funnel plots contributed to assess the likely presence of publication bias. Meta-analyses were developed in the software Review Manager (RevMan; The Nordic Cochrane Centre, The Cochrane Collaboration, Copenhagen, Denmark) version 5.3.

\section{Results}

In total, we identified 8,825 articles. After removal of 1,758 duplicates, two reviewers (K.V.F.A., R.A.S.), working independently, screened 7,067 titles with $\mathrm{k}=0.1907$. Subsequently, 161 abstracts of the selected titles were independently screened by the same reviewers (with $\mathrm{k}=0.4385$ ), retaining 40 articles for full-text review.

In hand searches, the reviewers did not find eligible articles. One additional article was added from the reference lists examined in the full-text review. Finally, 25 articles were retained for qualitative synthesis and nine RCTs for meta-analysis (Figure 1).

\section{Figure 1}

PRISMA flow diagram for systematic review and meta-analysis.

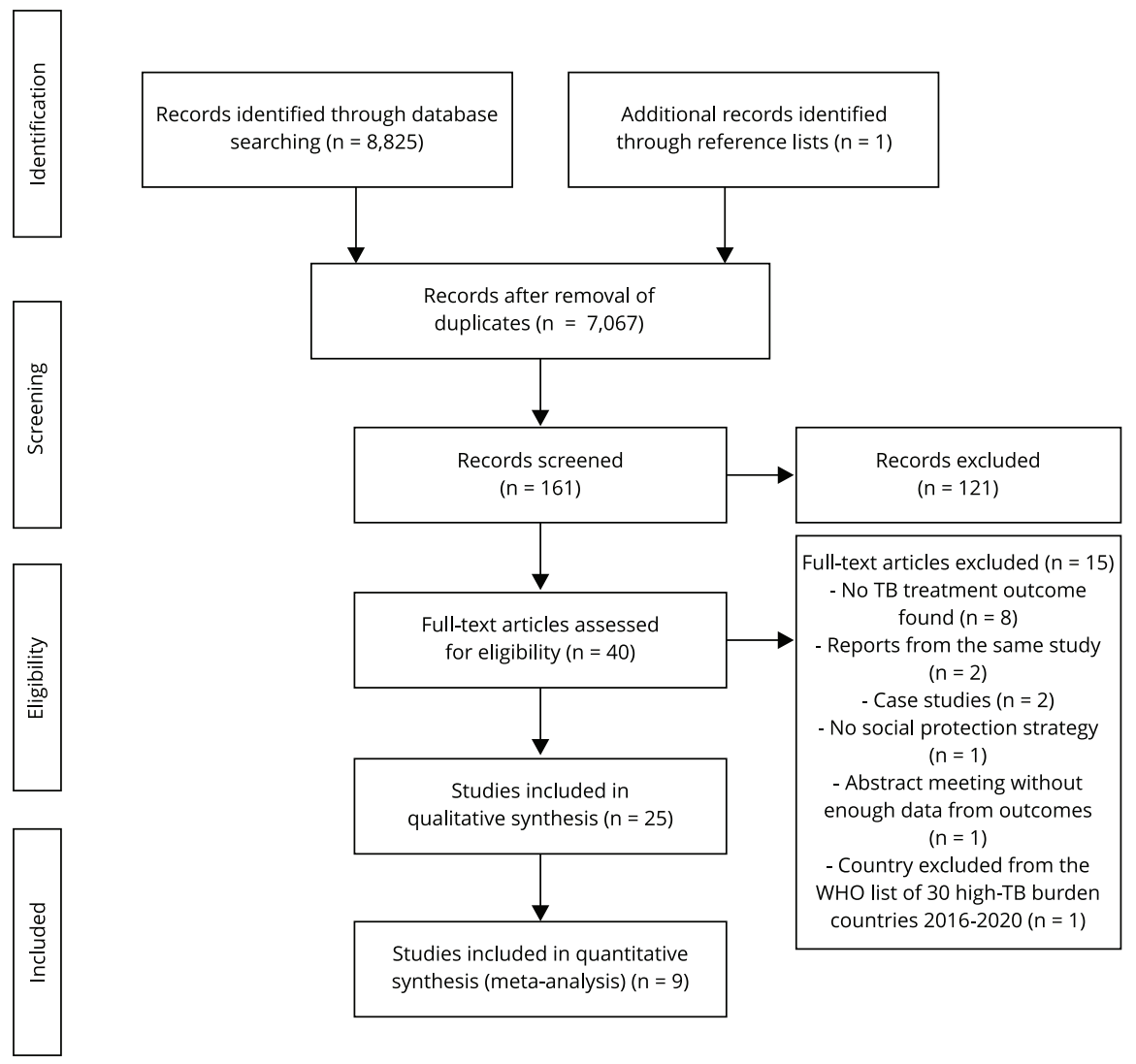

WHO: World Health Organization. 


\section{Description of studies included in qualitative synthesis $(n=25)$}

Nineteen studies were conducted in middle-income countries (13 in upper-middle and six in lower-middle). Four studies were developed in three low-income countries: Ethiopia 19,20, Malawi 21 and Nepal 22. Two studies 23,24 were developed in Russia, which is not in low- and middle-income categories, but it is listed between the high TB-burden countries 2 .

Seventeen studies were performed in high TB-burden countries. The other studies $(n=8)$ occurred in countries out of this category, but referred to as low-income: Malawi 21 and Nepal 22 or middle-income countries: Ecuador 25, Mexico 26, Republic of Moldova 27, Peru 28, Swaziland 29 and Timor-Leste 30.

Quasi-experimental designs $(\mathrm{n}=11)$ accounted for most of the reviewed studies, followed by RCTs $(n=9)$ and observational studies $(n=5)$. Seventeen studies (among then eight RCTs) presented evidences on effects of non-financial interventions for TB patients (lay community health workers or social workers $19,28,31,32,33,34,35$, food assistance $23,27,28,30,36,37$, counselling 22 , "TB clubs" 20 , training programs 26 , social franchising 38 , socio-educational approaches 21 ).

Twelve studies presented patient or treatment supporters directed financial interventions (among then seven quasi-experimental studies), including monetary incentives $22,24,25,27,28,29,35,39,40,41$, a conditional cash transfer program 42 and economic support by voucher delivery ${ }^{43}$. Some studies $22,27,28,35$ combined financial and non-financial interventions.

Sixteen studies were prospective with primary data collection and nine studies used secondary data sources. Seventeen studies did not include TB vulnerable populations. Eleven studies only included individuals with pulmonary TB and eight did not restrict TB clinical forms. See Table 1 for details of this section.

\section{Description of TB treatment outcomes}

\section{- $\quad$ Treatment success rates (TSR)}

Twenty-two controlled studies showed TSR for TB patients. In quasi-experimental studies ( $\mathrm{n}=$ 10), TSR ranged from 69.3 to $96.9 \%$ in intervention groups and from 31.1 to $96.9 \%$ in controls. In RCTs $(\mathrm{n}=9$ ), TSR ranged from 70 to $97.7 \%$ in intervention groups and from 57.5 to $84.1 \%$ in controls. In observational studies $(\mathrm{n}=3)$, TSR ranged from 68.7 to $95.8 \%$ in individuals exposed to social protection and from 46.9 to $92.9 \%$ in non-exposed individuals. The largest difference in TSR between study groups (47\%) was found in a quasi-experimental study conducted in Saint Petersburg, Russian Federation 23.

Few studies did not show effects of SPS on TB treatment outcomes. In a RCT conducted in Dili, Timor-Leste 30 food incentives did not significantly improve these outcomes. A study 40 that assessed effects of transportation incentives in China did not detect differences in TSR. Pragmatic RCT developed in South Africa 43 showed a small but non-significant improvement in TSR after providing economic support to TB patients.

\section{- Cure rates (CR)}

CR of TB patients were presented by 13 studies. In RCTs $(n=8)$ intervention groups achieved higher CR (ranging from 26.7 to $97.7 \%$ ) than in controls (ranging from 10 to $81.4 \%$ ). In quasi-experimental studies $(n=4)$ intervention groups also achieved higher CR (ranging from 11.8 to $82.4 \%$ ) than in controls (ranging from 0 to $74.8 \%$ ). Among observational studies, only one presented CR (equals to $82.1 \%$ in exposed and $76.9 \%$ in non-exposed individuals).

\section{- Treatment default rates}

Twenty studies presented default rates for TB treatment. Quasi-experimental studies $(\mathrm{n}=10)$ showed proportions of default in intervention groups ranging from $0.2-21.8 \%$ and in controls from 0.1 $68.9 \%$. In RCTs $(n=7)$ proportions of default in individuals exposed to social protection ranged from 
Table 1

Summary of all reviewed studies.

\begin{tabular}{|c|c|c|c|c|c|c|c|}
\hline $\begin{array}{l}\text { Study/ } \\
\text { Country }\end{array}$ & $\begin{array}{l}\text { Income/TB- } \\
\text { burden }\end{array}$ & $\mathbf{n}$ & $\begin{array}{l}\text { Study } \\
\text { location }\end{array}$ & $\begin{array}{l}\text { Study design } \\
\text { (score)* }\end{array}$ & $\begin{array}{l}\text { Population and } \\
\text { study period }\end{array}$ & Intervention & Main outcome \\
\hline $\begin{array}{l}\text { Baral et al. 22/ } \\
\text { Nepal }\end{array}$ & Low-income & $\begin{array}{l}\mathrm{I}_{1}: 33 \\
\mathrm{I}_{2}: 42 \\
\mathrm{C}: 81\end{array}$ & $\begin{array}{l}7 \text { DOTS-plus } \\
\text { centers }\end{array}$ & $\begin{array}{l}\text { Mixed-method } \\
\text { (intervention } \\
\text { and qualitative) }\end{array}$ & $\begin{array}{l}\text { MDR-TB patients } \\
\text { (Jan-Dec 2008) }\end{array}$ & $\begin{array}{l}\mathrm{I}_{1}: \text { counselling. } \\
\mathrm{I}_{2} \text { : counselling } \\
\text { and financial } \\
\text { support }\end{array}$ & $\begin{array}{c}\text { Cure }\left(I_{1}: 85 \% ; I_{2}\right. \\
76 \% ; C: 67 \%)\end{array}$ \\
\hline $\begin{array}{l}\text { Cantalice } \\
\text { Filho 37/Brazil }\end{array}$ & $\begin{array}{l}\text { Upper } \\
\text { middle- } \\
\text { income } \\
\text { High TB- } \\
\text { burden }\end{array}$ & $\begin{array}{c}142 \\
\text { (I: 74, C: 68) }\end{array}$ & $\begin{array}{l}\text { Primary care } \\
\text { clinics in } \\
\text { Duque de } \\
\text { Caxias, Rio } \\
\text { de Janeiro } \\
\text { State }\end{array}$ & $\begin{array}{l}\text { Retrospective } \\
\text { comparative } \\
\text { study (7 points) }\end{array}$ & $\begin{array}{l}\geq 15 \text { years old } \\
\text { with confirmed } \\
\text { TB diagnosis } \\
\text { (Jan 2004-Jul } \\
\text { 2006) }\end{array}$ & $\begin{array}{c}\text { Monthly } \\
\text { food baskets } \\
\text { delivered in } \\
\text { the healthcare } \\
\text { clinic }\end{array}$ & $\begin{array}{c}\text { Cure (I: 87.1\%; } \\
\text { C: } 69.7 \%)\end{array}$ \\
\hline $\begin{array}{l}\text { Ciobanu et al. } \\
\text { 27/Republic of } \\
\text { Moldova }\end{array}$ & $\begin{array}{l}\text { Lower } \\
\text { middle- } \\
\text { income }\end{array}$ & $\begin{array}{c}4,870 \text { (I: } 2378 \\
\text { C: } 2492)\end{array}$ & $\begin{array}{l}\text { National } \\
\text { data, before } \\
\text { (2008) and } \\
\text { after (2011) } \\
\text { incentives }\end{array}$ & $\begin{array}{c}\text { Retrospective } \\
\text { cohort study (14 } \\
\text { points) }\end{array}$ & $\begin{array}{l}\geq 18 \text { years old, } \\
\text { TB patients } \\
\text { treated in } 2008 \\
\text { and in } 2011\end{array}$ & $\begin{array}{l}\text { Cash, non- } \\
\text { cash or both } \\
\text { incentives. } \\
\text { (I: groups that } \\
\text { received cash) }\end{array}$ & $\begin{array}{l}\text { TSR (I: } 88 \% ; \\
\text { C: } 79 \% ; \\
\text { p < 0.001) }\end{array}$ \\
\hline $\begin{array}{l}\text { Clarke et } \\
\text { al. 31/South } \\
\text { Africa }\end{array}$ & $\begin{array}{l}\text { Upper } \\
\text { middle- } \\
\text { income } \\
\text { High TB- } \\
\text { burden }\end{array}$ & $\begin{array}{c}89 \text { (I: 47, } \\
\text { C: } 42)\end{array}$ & $\begin{array}{l}211 \text { farms (I: } \\
\text { 106; C: 105) }\end{array}$ & $\begin{array}{c}\text { Cluster } \\
\text { randomized } \\
\text { controlled trial }\end{array}$ & $\begin{array}{l}\text { Permanent } \\
\text { farm dwellers } \\
\geq 15 \text { years old } \\
\text { treated (from } \\
\text { Nov } 1,2000 \text { to } \\
\text { Oct } 31,2001 \text { ) }\end{array}$ & $\begin{array}{l}\text { Adult farm } \\
\text { dwellers } \\
\text { trained as lay } \\
\text { health workers }\end{array}$ & $\begin{array}{l}\text { TSR (I: 83\%; } \\
\text { C: } 64.3 \% ; \\
p=0.042)\end{array}$ \\
\hline $\begin{array}{l}\text { Datiko \& } \\
\text { Lindtjørn 19/ } \\
\text { Ethiopia }\end{array}$ & $\begin{array}{l}\text { Low-income } \\
\text { High TB- } \\
\text { burden }\end{array}$ & $\begin{array}{c}318 \text { (I: } 230 \\
\text { C: } 88)\end{array}$ & $\begin{array}{l}51 \text { kebeles } \\
\text { in two rural } \\
\text { districts of } \\
\text { Southern } \\
\text { Ethiopia }\end{array}$ & $\begin{array}{l}\text { Community- } \\
\text { randomized trial }\end{array}$ & $\begin{array}{l}\text { All new smear- } \\
\text { positive } \\
\text { pulmonary TB } \\
\text { cases }\end{array}$ & $\begin{array}{c}\text { Trained } \\
\text { community } \\
\text { health workers }\end{array}$ & $\begin{array}{c}\text { TSR (I: 89.3\%; } \\
\text { C: } 81.3 \% \\
\text { p = 0.012) }\end{array}$ \\
\hline $\begin{array}{l}\text { Demissie et } \\
\text { al. 20/Ethiopia }\end{array}$ & $\begin{array}{l}\text { Low-income } \\
\text { High TB- } \\
\text { burden }\end{array}$ & $\begin{array}{c}128 \text { (I: 64, } \\
\text { C: } 64)\end{array}$ & $\begin{array}{l}2 \text { rural } \\
\text { districts of } \\
\text { Northern } \\
\text { Ethiopia }\end{array}$ & $\begin{array}{l}\text { Mixed-method } \\
\text { - cohort and } \\
\text { qualitative (12 } \\
\text { points) }\end{array}$ & $\begin{array}{l}\text { Smear-positive } \\
\text { TB patients } \\
\text { (from July } 1 \text { to } \\
\text { Oct } 15,1998 \text { ) }\end{array}$ & $\begin{array}{l}\text { TB patients in } \\
\text { rural kebeles } \\
\text { organized in } \\
\text { "TB clubs" }\end{array}$ & $\begin{array}{c}\text { TCR (I: } 68.7 \% \\
\text { C: } 46.8 \% \\
\text { p }=0.02)\end{array}$ \\
\hline $\begin{array}{l}\text { Gärden et } \\
\text { al. 23/Russia }\end{array}$ & $\begin{array}{l}\text { High-income } \\
\text { High TB- } \\
\text { burden }\end{array}$ & $\begin{array}{c}518 \text { (I: } 142 \\
\text { C: } 376)\end{array}$ & $\begin{array}{c}\text { St. } \\
\text { Petersburg's } \\
\text { TB } \\
\text { dispensary }\end{array}$ & $\begin{array}{c}\text { Historical } \\
\text { controlled } \\
\text { intervention } \\
\text { study (11 points) }\end{array}$ & $\begin{array}{l}\text { Homeless } \\
\text { patients referred } \\
\text { to TB dispensary } \\
\text { (from Dec 2001 } \\
\text { to Jan 2004) }\end{array}$ & $\begin{array}{l}\text { Food packages } \\
\text { delivered once } \\
\text { a day } 5 \text { days } \\
\text { a week and } \\
\text { support from a } \\
\text { social worker }\end{array}$ & $\begin{array}{c}\text { TSR (I: } 78.2 \% \\
\text { C: } 31.0 \%)\end{array}$ \\
\hline $\begin{array}{l}\text { Jakubowiak et } \\
\text { al. 24/Russia }\end{array}$ & $\begin{array}{l}\text { High-income } \\
\text { High TB- } \\
\text { burden }\end{array}$ & $\begin{array}{c}\text { 1,389 (I: 382, } \\
\text { C: } 1,007)\end{array}$ & $\begin{array}{l}4 \text { regions } \\
\text { with TB } \\
\text { services }\end{array}$ & $\begin{array}{l}\text { Cross-sectional } \\
\text { study ( } 5 \text { points) }\end{array}$ & $\begin{array}{l}\text { New pulmonary } \\
\text { TB patients } \geq \\
15 \text { years old } \\
\text { treated (from } \\
\text { Jan } 1,2004 \text { to } \\
\text { Mar } 31,2005 \text { ) }\end{array}$ & $\begin{array}{c}\text { Social support } \\
\text { during TB } \\
\text { treatment } \\
\text { (food packs, } \\
\text { hygiene kits, } \\
\text { transportation } \\
\text { incentives, } \\
\text { etc.) }\end{array}$ & $\begin{array}{c}28.1 \% \text { of } \\
\text { adherents and } \\
18.4 \% \text { of non- } \\
\text { adherents with } \\
\text { incentives }\end{array}$ \\
\hline $\begin{array}{l}\text { Kliner et al. 29/ } \\
\text { Swaziland }\end{array}$ & $\begin{array}{l}\text { Lower } \\
\text { middle- } \\
\text { income }\end{array}$ & $\begin{array}{c}\text { 1,077 (I: 161, } \\
\text { C: } 916)\end{array}$ & $\begin{array}{l}\text { Hospital in a } \\
\text { rural district }\end{array}$ & $\begin{array}{c}\text { Pragmatic } \\
\text { controlled } \\
\text { interventional } \\
\text { study ( } 12 \text { points) }\end{array}$ & $\begin{array}{l}\text { TB patients (Jan } \\
\text { 2010-Sep 2011) }\end{array}$ & $\begin{array}{l}\text { Treatment } \\
\text { support from } \\
\text { community } \\
\text { workers }\end{array}$ & $\begin{array}{c}\text { TSR (I: } 73 \% \text { vs. } \\
\text { C: } 60 \% \\
p=0.003)\end{array}$ \\
\hline
\end{tabular}

(continues) 
Table 1 (continued)

\begin{tabular}{|c|c|c|c|c|c|c|c|}
\hline $\begin{array}{l}\text { Study/ } \\
\text { Country }\end{array}$ & $\begin{array}{l}\text { Income/TB- } \\
\text { burden }\end{array}$ & $\mathbf{n}$ & $\begin{array}{l}\text { Study } \\
\text { location }\end{array}$ & $\begin{array}{l}\text { Study design } \\
\text { (score)* }\end{array}$ & $\begin{array}{l}\text { Population and } \\
\text { study period }\end{array}$ & Intervention & Main outcome \\
\hline $\begin{array}{l}\text { Lönnroth } \\
\text { et al. 38/ } \\
\text { Myanmar }\end{array}$ & $\begin{array}{l}\text { Lower } \\
\text { middle- } \\
\text { income } \\
\text { High TB- } \\
\text { burden }\end{array}$ & $\begin{array}{l}253 \text { (non- } \\
\text { controlled) }\end{array}$ & $\begin{array}{l}\text { National case } \\
\text { notification } \\
\text { data and } \\
\text { survey in } \\
\text { clinics in } \\
\text { Yangon }\end{array}$ & $\begin{array}{l}\text { Cross-sectional } \\
\text { study (10 points) }\end{array}$ & $\begin{array}{l}\text { All patients } \\
\text { treated in Sun } \\
\text { Quality Health } \\
\text { (SQH) clinics in } \\
\text { Yangon (from } \\
\text { Sep } 1 \text { to Oct } 30 \\
\text { 2004) }\end{array}$ & $\begin{array}{l}\text { Sun Quality } \\
\text { Health (SQH), a } \\
\text { social franchise } \\
\text { that licensed } \\
\text { practitioners } \\
\text { with clinics } \\
\text { serving low- } \\
\text { income people }\end{array}$ & $\begin{array}{c}\text { TSR for new } \\
\text { smear-positive } \\
\text { cases was 84\% }\end{array}$ \\
\hline $\begin{array}{l}\text { Lutge et al. } 43 / \\
\text { South Africa }\end{array}$ & $\begin{array}{l}\text { Upper } \\
\text { middle- } \\
\text { income } \\
\text { High TB- } \\
\text { burden }\end{array}$ & $\begin{array}{c}4,091 \text { (I: } 2,107 \\
\text { C: } 1,984)\end{array}$ & $\begin{array}{c}20 \text { public } \\
\text { sector clinics } \\
\text { in Kwazulu- } \\
\text { Natal }\end{array}$ & $\begin{array}{l}\text { Cluster- } \\
\text { randomized } \\
\text { controlled trial }\end{array}$ & $\begin{array}{c}\text { TB patients } \\
\text { within (July } 1 \text {, } \\
2009 \text { to Mar 31, } \\
\text { 2010) }\end{array}$ & $\begin{array}{l}\text { Monthly } \\
\text { vouchers (US\$ } \\
\text { 15) redeemable } \\
\text { at specific } \\
\text { general stores }\end{array}$ & $\begin{array}{c}\text { TSR (I: } 76.2 \% \text { vs. } \\
\text { C: } 70.7 \% ; \\
p=0.107)\end{array}$ \\
\hline $\begin{array}{l}\text { Martins et } \\
\text { al. 30/Timor- } \\
\text { Leste }\end{array}$ & $\begin{array}{l}\text { Lower- } \\
\text { middle } \\
\text { income }\end{array}$ & $\begin{array}{c}265 \text { (I: } 136, \\
\text { C: } 129)\end{array}$ & $\begin{array}{c}3 \text { primary } \\
\text { clinics in Dili: } \\
\text { government, } \\
\text { private } \\
\text { and church } \\
\text { operated) }\end{array}$ & $\begin{array}{l}\text { Randomized } \\
\text { controlled trial }\end{array}$ & $\begin{array}{l}\text { TB patients } \\
\text { aged } \geq 18 \text { and } \\
\text { that agree to } \\
\text { treatment at } \\
\text { diagnostic clinic } \\
\text { for eight months }\end{array}$ & $\begin{array}{c}\text { Daily meal in } \\
\text { attendance to } \\
\text { the clinic and } \\
\text { unprepared } \\
\text { food to take } \\
\text { home, in } \\
\text { continuation } \\
\text { phase }\end{array}$ & $\begin{array}{l}\text { TSR (I: 76\% vs. } \\
\text { C: } 78 \% ; p=0.7)\end{array}$ \\
\hline $\begin{array}{l}\text { Ngamvithaya- } \\
\text { pong-Yanai et } \\
\text { al. 35/Thailand }\end{array}$ & $\begin{array}{l}\text { Upper } \\
\text { middle- } \\
\text { income } \\
\text { High TB- } \\
\text { burden }\end{array}$ & $\begin{array}{c}759 \text { (I: } 192 \\
\text { C: } 567)\end{array}$ & $\begin{array}{l}\text { Chiang Rai, } \\
\text { Thailand's } \\
\text { northern } \\
\text { province }\end{array}$ & $\begin{array}{l}\text { Intervention } \\
\text { study - before } \\
\text { and after (7 } \\
\text { points) }\end{array}$ & $\begin{array}{l}\text { Extremely poor } \\
\text { TB patients, } \\
\text { living alone, } \\
\text { with elderly } \\
\text { caregivers or } \\
\text { isolated from } \\
\text { community }\end{array}$ & $\begin{array}{l}\text { Engagement } \\
\text { of Chiang } \\
\text { Rai women's } \\
\text { organization to } \\
\text { support them } \\
\text { financially and } \\
\text { socially }\end{array}$ & $\begin{array}{c}\text { TSR (I: } 69.3 \% \text { vs. } \\
\text { C: } 51.6 \% \\
\text { p < } 0.00)\end{array}$ \\
\hline $\begin{array}{l}\text { Ritchie et } \\
\text { al. 21/Malawi }\end{array}$ & Low-income & $\begin{array}{c}110 \text { (I: } 30 \\
\text { C: } 80)\end{array}$ & $\begin{array}{l}28 \text { health } \\
\text { centers } \\
\text { in Zomba } \\
\text { district }\end{array}$ & $\begin{array}{c}\text { Cluster } \\
\text { randomized } \\
\text { controlled trial }\end{array}$ & $\begin{array}{l}\text { All lay health } \\
\text { workers } \\
\text { involved in } \\
\text { providing care } \\
\text { to TB patients }\end{array}$ & $\begin{array}{l}\text { Two knowledge } \\
\text { translation } \\
\text { interventions: } \\
\text { educational } \\
\text { outreach and } \\
\text { reminders }\end{array}$ & $\begin{array}{c}\text { TSR (I: } 70 \% \text { vs. } \\
\text { C: } 58 \% \\
p=0.578)\end{array}$ \\
\hline $\begin{array}{l}\text { Rocha } \\
\text { et al. 28/Peru }\end{array}$ & $\begin{array}{l}\text { Upper } \\
\text { middle- } \\
\text { income }\end{array}$ & $\begin{array}{c}\text { 1,861 (I: } 307 \\
\text { C: } 1,554)\end{array}$ & $\begin{array}{l}\text { Eight } \\
\text { contiguous } \\
\text { slums in } \\
\text { Northern } \\
\text { Lima }\end{array}$ & $\begin{array}{l}\text { Intervention } \\
\text { study (4 points) }\end{array}$ & $\begin{array}{l}\text { Subsequently } \\
\text { diagnosed TB } \\
\text { patients and } \\
\text { their household } \\
\text { contacts (Dec } \\
\text { 2007-Oct 2010) }\end{array}$ & $\begin{array}{l}\text { Household visits, } \\
\text { counselling, } \\
\text { food and cash } \\
\text { transfers, } \\
\text { microenterprise, } \\
\text { microcredits and } \\
\text { training }\end{array}$ & $\begin{array}{l}\text { TSR ( } 91 \% \\
\text { before vs. } \\
\text { 97\% after } \\
\text { intervention) }\end{array}$ \\
\hline $\begin{array}{l}\text { Singh } \\
\text { et al. 32/India }\end{array}$ & $\begin{array}{l}\text { Lower } \\
\text { middle- } \\
\text { income } \\
\text { High TB- } \\
\text { burden }\end{array}$ & $\begin{array}{c}617 \text { (I:1 41, } \\
\text { C: } 476)\end{array}$ & $\begin{array}{c}\text { One } \\
\text { tuberculosis } \\
\text { unit covering } \\
\text { a population } \\
\text { of } 600,000 \\
\text { in Haryana } \\
\text { State }\end{array}$ & $\begin{array}{l}\text { Intervention } \\
\text { study (10 points) }\end{array}$ & $\begin{array}{l}\text { New sputum } \\
\text { smear-positive } \\
\text { patients } \\
\text { registered in the } \\
\text { tuberculosis unit } \\
\text { for treatment }\end{array}$ & $\begin{array}{c}\text { Directly } \\
\text { Observed } \\
\text { Treatment (DOT) } \\
\text { from community } \\
\text { volunteers vs. } \\
\text { government } \\
\text { health workers }\end{array}$ & $\begin{array}{c}\text { TSR (I: } 78 \% \text { vs. } \\
\text { C: } 77 \%)\end{array}$ \\
\hline
\end{tabular}

(continues) 
Table 1 (continued)

\begin{tabular}{|c|c|c|c|c|c|c|c|}
\hline $\begin{array}{l}\text { Study/ } \\
\text { Country }\end{array}$ & $\begin{array}{l}\text { Income/TB- } \\
\text { burden }\end{array}$ & $\mathbf{n}$ & $\begin{array}{l}\text { Study } \\
\text { location }\end{array}$ & $\begin{array}{l}\text { Study design } \\
\text { (score)* }\end{array}$ & $\begin{array}{l}\text { Population and } \\
\text { study period }\end{array}$ & Intervention & Main outcome \\
\hline $\begin{array}{l}\text { Soares } \\
\text { et al. 33/Brazil }\end{array}$ & $\begin{array}{l}\text { Upper } \\
\text { middle- } \\
\text { income } \\
\text { High TB- } \\
\text { burden }\end{array}$ & $\begin{array}{c}2,623 \text { (I: } 1,771 \\
\text { C: } 852)\end{array}$ & $\begin{array}{l}\text { Rocinha } \\
\text { Favela, } \\
\text { the largest } \\
\text { urban slum } \\
\text { in South } \\
\text { America }\end{array}$ & $\begin{array}{l}\text { Intervention } \\
\text { study (before } \\
\text { and after) (13 } \\
\text { points) }\end{array}$ & $\begin{array}{l}\text { All patients with } \\
\text { pulmonary or } \\
\text { extra-pulmonary } \\
\text { TB who started } \\
\text { treatment } \\
\text { between } 2001 \\
\text { and } 2008\end{array}$ & $\begin{array}{l}\text { DOT implemen } \\
\text { tation and } \\
\text { training } 40 \text { lay } \\
\text { persons as } \\
\text { community } \\
\text { health } \\
\text { workers }\end{array}$ & $\begin{array}{l}\text { TSR }(83.2 \% \\
\text { vs. } 67.6 \% ; \\
\text { p }<0.001) \\
\text { pre- and post- } \\
\text { intervention }\end{array}$ \\
\hline $\begin{array}{l}\text { Sripad } \\
\text { et al. 25/ } \\
\text { Ecuador }\end{array}$ & $\begin{array}{l}\text { Upper } \\
\text { middle- } \\
\text { income }\end{array}$ & $\begin{array}{l}191 \text { (I: 105, } \\
\text { C: } 86)\end{array}$ & $\begin{array}{l}\text { Ecuador's } \\
\text { NTP }\end{array}$ & $\begin{array}{l}\text { Non- } \\
\text { randomized trial } \\
\text { with historical } \\
\text { controls (8 } \\
\text { points) }\end{array}$ & $\begin{array}{c}\text { Drug-resistant } \\
\text { (DR-TB) patients } \\
\text { (from Aug } 2011 \\
\text { to Jan } 2012 \text { - } \\
\text { intervention } \\
\text { and from Jan to } \\
\text { Aug } 2010 \text { - pre- } \\
\text { program) }\end{array}$ & $\begin{array}{c}\text { Ecuador's } \\
\text { NTP enacted } \\
\text { a monetary } \\
\text { incentive } \\
\text { program giving } \\
\text { adherent DR- } \\
\text { TB patients } \\
\text { a USD } 240 \\
\text { bonus each } \\
\text { month }\end{array}$ & $\begin{array}{l}\text { 1-year default } \\
\text { rate }(9.5 \% \\
\text { vs. } 26.7 \% ; \\
\text { p }<0.05) \text {, in } \\
\text { program and } \\
\text { pre-program }\end{array}$ \\
\hline $\begin{array}{l}\text { Sudarsanam } \\
\text { et al. } 36 / \text { India }\end{array}$ & $\begin{array}{l}\text { Lower } \\
\text { middle- } \\
\text { income } \\
\text { High TB- } \\
\text { burden }\end{array}$ & $\begin{array}{c}97 \text { (I: 48, } \\
\text { C: } 49)\end{array}$ & $\begin{array}{l}\text { One of four } \\
\text { clinics in } \\
\text { Vellore town, } \\
\text { southern } \\
\text { Indian state } \\
\text { of Tamil } \\
\text { Nadu }\end{array}$ & $\begin{array}{l}\text { Randomized } \\
\text { controlled trial }\end{array}$ & $\begin{array}{l}\text { Patients aged } \\
>12 \text { years } \\
\text { diagnosed with } \\
\text { TB (recruited } \\
\text { between Jan and } \\
\text { Nov 2005) }\end{array}$ & $\begin{array}{l}\text { Macronutrient } \\
\text { supplement } \\
\text { (cereal } \\
\text { and lentil } \\
\text { mixture) and } \\
\text { micronutrients } \\
\text { (one-a-day } \\
\text { multivitamin } \\
\text { table) }\end{array}$ & $\begin{array}{l}\text { Higher poor } \\
\text { outcomes } \\
\text { in the non- } \\
\text { supplemented } \\
\text { HIV-TB co- } \\
\text { infected group }\end{array}$ \\
\hline $\begin{array}{l}\text { Torrens } \\
\text { et al. 42/Brazil }\end{array}$ & $\begin{array}{l}\text { Upper } \\
\text { middle- } \\
\text { income } \\
\text { High TB- } \\
\text { burden }\end{array}$ & $\begin{array}{c}7,255 \text { (I: 5,788, } \\
\text { C: } 1,467)\end{array}$ & $\begin{array}{l}\text { Brazilian } \\
\text { national } \\
\text { databases } \\
\text { (SINAN and } \\
\text { CadÚnico) }\end{array}$ & $\begin{array}{c}\text { Retrospective } \\
\text { cohort (14 } \\
\text { points) }\end{array}$ & $\begin{array}{l}\text { All new TB cases } \\
\text { diagnosed in } \\
\text { 2010, recorded } \\
\text { in SINAN } \\
\text { database and } \\
\text { registered in } \\
\text { Cadúnico }\end{array}$ & $\begin{array}{l}\text { Brazilian } \\
\text { national } \\
\text { conditional } \\
\text { cash transfer } \\
\text { (Brazilian } \\
\text { Income } \\
\text { Transfer } \\
\text { Program) }\end{array}$ & $\begin{array}{c}\text { Cure rates } \\
\text { (I: } 82.1 \% \text { vs. } \\
\text { C: } 76.9 \% ; \\
\text { p < 0.001) }\end{array}$ \\
\hline $\begin{array}{l}\text { Wei et al. 39/ } \\
\text { China }\end{array}$ & $\begin{array}{l}\text { Upper } \\
\text { middle- } \\
\text { income } \\
\text { High TB- } \\
\text { burden }\end{array}$ & $\begin{array}{c}183 \text { (I: 90, } \\
\text { C: } 93)\end{array}$ & $\begin{array}{c}2 \text { districts of } \\
\text { Shanghai (1 } \\
\text { interven } \\
\text { tion/1 } \\
\text { control) }\end{array}$ & $\begin{array}{c}\text { Controlled } \\
\text { intervention } \\
\text { study - before } \\
\text { and after (8 } \\
\text { points) }\end{array}$ & $\begin{array}{l}\text { Poor migrants } \\
\text { TB patients }\end{array}$ & $\begin{array}{c}\text { Financial } \\
\text { incentives to } \\
\text { poor migrant } \\
\text { TB patients } \\
\text { (transportation } \\
\text { and living } \\
\text { subsidies) }\end{array}$ & $\begin{array}{l}\text { TCR (I: from } \\
78 \% \text { to } 89 \% ; \\
\text { and C: from } \\
73 \% \text { to } 76 \% ; \\
\text { p = 0.03) }\end{array}$ \\
\hline $\begin{array}{l}\text { Yao et al. 40/ } \\
\text { China }\end{array}$ & $\begin{array}{l}\text { Upper } \\
\text { middle- } \\
\text { income } \\
\text { High TB- } \\
\text { burden }\end{array}$ & $\begin{array}{c}9,194 \text { (I: 5,449, } \\
\text { C: } 3,745)\end{array}$ & $\begin{array}{l}\text { Fifty poor } \\
\text { counties } \\
\text { of Shanxi } \\
\text { (Fidelis } \\
\text { project) and } \\
51 \text { control } \\
\text { counties }\end{array}$ & $\begin{array}{l}\text { Pilot evaluation } \\
\text { study (8 points) }\end{array}$ & $\begin{array}{l}\text { New TB cases } \\
\text { in baseline } \\
\text { (Jan-Sep 2004) } \\
\text { and during the } \\
\text { intervention } \\
\text { (Jan-Sep 2005) } \\
\text { using routine TB } \\
\text { reporting data }\end{array}$ & $\begin{array}{c}\text { Financial } \\
\text { incentives } \\
\text { for doctors. } \\
\text { Incentives to } \\
\text { village leaders } \\
\text { for community } \\
\text { health } \\
\text { education }\end{array}$ & $\begin{array}{l}\text { TSR baseline } \\
\text { (I: } 95.3 \text { vs. C: } \\
\text { 93.9\%; p < } \\
\text { 0.01); project } \\
\text { (I: } 96.9 \text { vs. C: } \\
\text { 96.9\%; p > 0.05) }\end{array}$ \\
\hline
\end{tabular}

(continues) 
Table 1 (continued)

\begin{tabular}{|c|c|c|c|c|c|c|c|}
\hline $\begin{array}{l}\text { Study/ } \\
\text { Country }\end{array}$ & $\begin{array}{l}\text { Income/TB- } \\
\text { burden }\end{array}$ & $\mathbf{n}$ & $\begin{array}{l}\text { Study } \\
\text { location }\end{array}$ & $\begin{array}{l}\text { Study design } \\
\text { (score)* }\end{array}$ & $\begin{array}{l}\text { Population and } \\
\text { study period }\end{array}$ & Intervention & Main outcome \\
\hline $\begin{array}{l}\text { Zou et al. 41/ } \\
\text { China }\end{array}$ & $\begin{array}{l}\text { Upper } \\
\text { middle- } \\
\text { income } \\
\text { High TB- } \\
\text { burden }\end{array}$ & $\begin{array}{c}356 \text { (I: } 263 \\
\text { C: } 93)\end{array}$ & $\begin{array}{c}\text { Three } \\
\text { districts } \\
\text { located in } \\
\text { Shanghai: (i) } \\
\text { Communi } \\
\text { cable Disease } \\
\text { Research } \\
\text { Consortium } \\
\text { (COMDIS), } \\
\text { (ii) The } \\
\text { Global Fund } \\
\text { Project, (iii) } \\
\text { control }\end{array}$ & $\begin{array}{l}\text { Intervention } \\
\text { study (case } \\
\text { study) - before } \\
\text { and after (8 } \\
\text { points) }\end{array}$ & $\begin{array}{l}\text { Poor migrants } \\
\text { TB patients } \\
\text { living in } \\
\text { Shanghai, China, } \\
\text { targeted by } \\
\text { two projects } \\
\text { involving } \\
\text { financial } \\
\text { incentives } \\
\text { (introduced in } \\
\text { Oct 2007) }\end{array}$ & $\begin{array}{l}\text { COMDIS: single } \\
\text { living and } \\
\text { transportation } \\
\text { subsidies } \\
\text { Global Fund } \\
\text { Project: } \\
\text { living and, } \\
\text { transportation } \\
\text { incentives, } \\
\text { plus incentives } \\
\text { to clinic staff } \\
\text { for each } \\
\text { extended } \\
\text { working hour }\end{array}$ & $\begin{array}{l}\text { TCR District I } \\
\text { (78 vs. 89\%); } \\
\text { District II (73 vs. } \\
\text { 88\%); Control } \\
\text { (73 vs. 76\%). }\end{array}$ \\
\hline $\begin{array}{l}\text { Zwarenstein } \\
\text { et al. 34/South } \\
\text { Africa }\end{array}$ & $\begin{array}{l}\text { Upper } \\
\text { middle- } \\
\text { income } \\
\text { High TB- } \\
\text { burden }\end{array}$ & $\begin{array}{l}98 \text { (I: } 54 \\
\text { C: } 44)\end{array}$ & $\begin{array}{l}\text { Four clinics } \\
\text { in Elsies } \\
\text { River, suburb } \\
\text { (20km from } \\
\text { Cape Town) }\end{array}$ & $\begin{array}{l}\text { Randomized } \\
\text { controlled trial }\end{array}$ & $\begin{array}{l}\text { Adult (aged } \\
>15 \text { years) } \\
\text { pulmonary } \\
\text { TB patients, } \\
\text { who started } \\
\text { TB treatment } \\
\text { (new and } \\
\text { retreatment) }\end{array}$ & $\begin{array}{l}\text { Supervision by } \\
\text { volunteers lay } \\
\text { health workers } \\
\text { in a poor } \\
\text { community/ } \\
\text { Supervision } \\
\text { by clinic } \\
\text { nurse/Self- } \\
\text { supervision }\end{array}$ & $\begin{array}{c}\% \text { of success: } \\
\text { lay health } \\
\text { workers (74\%), } \\
\text { clinic DOT } \\
(57 \%) \text { and self- } \\
\text { supervision } \\
(59 \%)\end{array}$ \\
\hline $\begin{array}{l}\text { Álvarez } \\
\text { Gordillo et } \\
\text { al. 26/Mexico }\end{array}$ & $\begin{array}{l}\text { Upper } \\
\text { middle- } \\
\text { income }\end{array}$ & $\begin{array}{l}87 \text { (I: } 44 \\
\text { C: } 43)\end{array}$ & $\begin{array}{c}\text { Health } \\
\text { centers in } \\
\text { Chiapas }\end{array}$ & $\begin{array}{l}\text { Controlled } \\
\text { intervention } \\
\text { study }\end{array}$ & $\begin{array}{c}\text { > } 15 \text { years old } \\
\text { with sputum } \\
\text { smear-positive } \\
\text { pulmonary TB } \\
\text { (Feb 2001-Jan } \\
\text { 2002) }\end{array}$ & $\begin{array}{c}\text { Training } \\
\text { program } \\
\text { for health } \\
\text { professionals } \\
\text { and self-help } \\
\text { groups for TB } \\
\text { patients }\end{array}$ & $\begin{array}{l}\text { TSR (I: } 97.7 \% ; \\
\text { C: } 81.4 \%)\end{array}$ \\
\hline
\end{tabular}

C: control group; CadÚnico: Unified Registry for Social Programmes; I: intervention group; NTP: National Tuberculosis Programme; SINAN: Notifiable Diseases Information System; TCR: treatment completion rates; TSR: treatment success rates.

* Quality scores with Downs \& Black 15 tool, except for randomized controlled trials - RCTs (in this review, we arbitrarily established that scores < 9 indicates bad quality evidences and $\geq 9$ indicates good quality evidences).

2.3-14.8\% and from 4.6-26.2\% in controls. Observational studies $(\mathrm{n}=3)$ showed lower default rates in exposed (4.2-12.5\%) than in non-exposed to SPS (7.0-40.6\%).

\section{- Treatment failure rates}

Twelve studies showed treatment failure rates. In RCTs $(n=6)$, they ranged from $0-8.5 \%$ in intervention groups and 0-9.5\% in controls. In quasi-experimental studies $(n=4)$, rates ranged from $0-5.6 \%$ in intervention groups and $0.1-1 \%$ in controls. Observational studies $(\mathrm{n}=2)$ showed failure rates ranging from $0-2.1 \%$ in intervention groups and $0-9.5 \%$ in controls.

\section{- Death rates}

Twenty studies showed death rates of TB patients. Among them, quasi-experimental $(\mathrm{n}=10)$, with values ranging from 0.2$21.8 \%$ in intervention groups and $0.1-68.9 \%$ in controls; RCTs $(\mathrm{n}=7)$ with values ranging from $2.3-14.8 \%$ in intervention groups and 4.6-26.2\% in controls. Finally, observational studies $(n=3)$ showed death rates ranging from $4.2-12.5 \%$ in intervention groups and $7-40.6 \%$ in controls. 


\section{- Quality assessment results}

The quality assessment with Downs \& Black checklist 15 showed a median score equal to 9 (with interquartile range from 7-12). The lowest score was four and the highest was 14 . Studies with the best quality evidences $(n=8)$ obtained scores greater than 9 (Table 1 ). The most important limitations of these studies were: lack of blinding of participants and study team, lack of information about follow-up lengths, and regarding the participants' compliance with study interventions. Lack of sample representativeness was also a common limitation in the studies.

\section{- $\quad$ Risk of bias assessment for RCTs}

Regarding randomization approach, all RCTs presented low risk of bias for random sequence generation and most of them (five studies) described an adequate allocation concealment. However, all RCTs were associated with high or unclear risk of bias due to lack of blinding of participants and study team. Outcome assessors were blinded in only one study. Incomplete outcome data and selective reporting did not account for relevant bias. Other sources of bias were associated with baseline imbalances, possibility of contamination across groups, low protocol fidelity and inadequate sample sizes (Figure 2).

According to GRADE approach ${ }^{17}$, evidences from most TB treatment outcomes presented serious limitations because of study design and execution (risk of bias). Only the outcome cure was downgraded once by inconsistency, which was classified as "serious" because of a high unexplained heterogeneity observation across studies (Table 2). Indirectness of evidences was observed for all outcomes. We downgraded evidences in one level by indirectness for both primary and secondary outcomes because of the diversity of interventions comprehended in the field of social protection and specific characteristics of participants in some studies (restricted to individuals with multidrug-resistant TB, participants with TB and HIV-TB coinfection, and homeless patients). Finally, imprecision did not account for serious limitations in the reviewed RCTs (Table 2).

Risk of bias assessment and visual inspection of funnel plots did not suggest the presence of selective reporting (publication bias). In addition, under the GRADE approach 17, the overall quality of evidences for effects of SPS on TB treatment outcomes was rated as low, except the evidences for cure (rated as very low quality).

\section{Meta-analysis}

The total number of participants in the RCTs included in meta-analysis $(\mathrm{n}=9)$ was 1,687 . These studies were conducted in seven countries, among them: South Africa 29,30,31, Ethiopia, India, Malawi, Mexico, Nepal and Timor-Leste. We separately performed meta-analyses according to each TB treatment outcome.

\section{- Primary outcome}

Pooled results of nine RCTs showed a significant association between SPS and TB treatment success $(\mathrm{RR}=1.09 ; 95 \% \mathrm{CI}: 1.03-1.14)$. Heterogeneity was not important among these studies $(\mathrm{I} 2=0 \% ; \mathrm{p}=$ 0.48) (Figure 3a).

\section{- Secondary outcomes}

In total, eight RCTs provided evidences for the cure of TB patients. A significant association between SPS and cure was found ( $R R=1.11 ; 95 \% \mathrm{CI}: 1.01-1.22)$. Additionally, substantial heterogeneity studies were not important $(\mathrm{I} 2=23 \% ; \mathrm{p}=0.25)$ as observed in Figure $3 \mathrm{~b}$.

Seven RCTs provided data regarding treatment default. A significant reduction in default was associated with SPS ( $R R=0.63$; 95\%CI: $0.45-0.89$ ), without evidences of important heterogeneity among studies ( $\mathrm{I}^{2}=0 \%, \mathrm{p}=0.67$ ) (Figure $3 \mathrm{c}$ ). Pooled RCTs also did not show an overall protective 


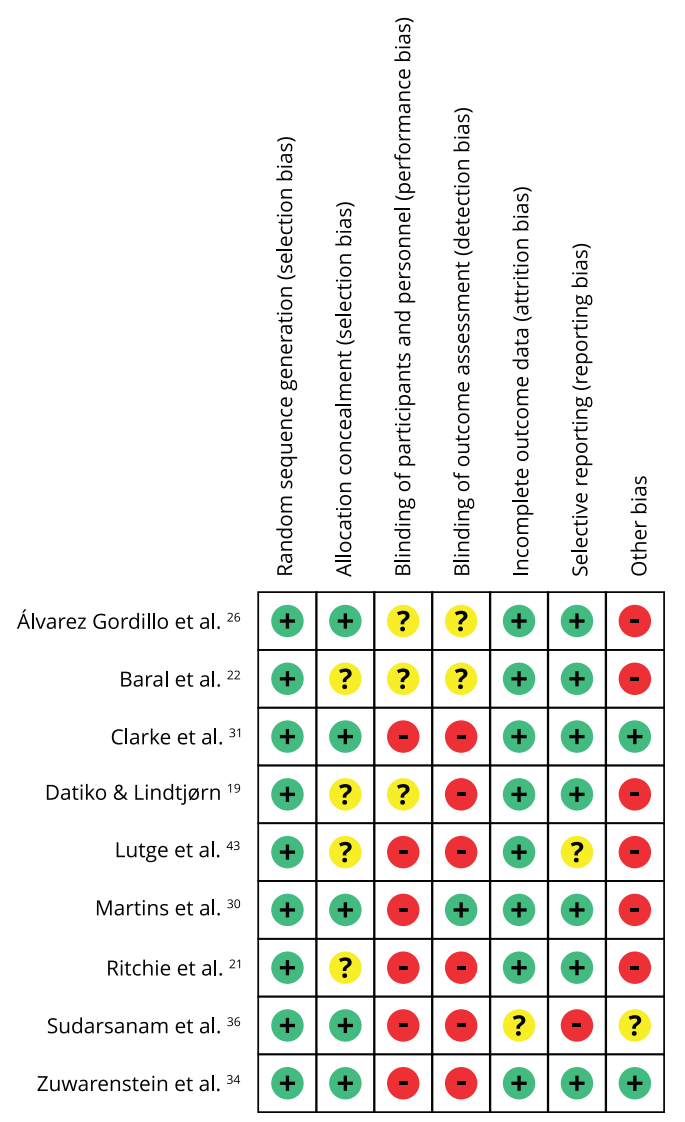

effect between SPS and treatment failure $(R R=0.78$; 95\%CI: 0.44-1.40), without evidences of heterogeneity $\left(\mathrm{I}^{2}=0 \%, \mathrm{p}=0.52\right)$ (Figure $\left.3 \mathrm{~d}\right)$.

Finally, overall risk ratio obtained from six RCTs showed that SPS was also not associated with TB death rates $(\mathrm{RR}=0.98$; 95\%CI: 0.61-1.57). Heterogeneity was also not detected among these studies $(\mathrm{I} 2=0 \%, \mathrm{p}=0.87)$ (Figure $3 \mathrm{e})$.

\section{Discussion}

This is the first systematic review focused on studies conducted in low and middle-income countries and/or with high TB-burden. We found an association between SPS and TB treatment success and a reduction in the risks of treatment default and therapeutic failure in patients under SPS. These evidences support the implementation of the social support along with a universal health coverage, especially in high-endemic TB or poor populations 1,8. However, our findings should be interpreted with caution, considering the low quality of evidences provided by current studies.

Among the reviewed studies, only one RCT developed in South Africa 43, showed that social protection did not improve the cure of TB patients. However, low fidelity to the trial protocol (leading to a third of eligible patients without intervention) and omission of data about HIV status of participants were important limitations to this study. 
Table 2

Summary of findings table.

\begin{tabular}{|c|c|c|c|c|c|}
\hline \multirow[t]{2}{*}{ Outcomes } & \multirow{2}{*}{$\begin{array}{l}\text { Number of participants } \\
\text { (studies) - follow-up }\end{array}$} & \multirow{2}{*}{$\begin{array}{l}\text { Quality of the evidence } \\
\text { (GRADE) }\end{array}$} & \multirow[t]{2}{*}{ RR $(95 \% \mathrm{Cl})$} & \multicolumn{2}{|c|}{ Anticipated absolute effects } \\
\hline & & & & $\begin{array}{l}\text { Risk with TB } \\
\text { usual care }\end{array}$ & $\begin{array}{l}\text { Risk difference with social } \\
\text { protection interventions }\end{array}$ \\
\hline Treatment success & 1,687 (9 RCTs) & $\oplus \oplus \bigcirc \bigcirc \operatorname{LOW} a, b$ & $1.09(1.03-1.14)$ & 723 per 1,000 & $\begin{array}{c}65 \text { more per } 1,000 \text { ( } 22 \text { more } \\
\text { to } 101 \text { more) }\end{array}$ \\
\hline Cure & 1,590 (8 RCTs) & $\oplus \bigcirc \bigcirc \bigcirc$ VERY LOW a,b,c & $1.11(1.01-1.22)$ & 493 per 1,000 & $\begin{array}{l}54 \text { more per } 1,000 \text { ( } 5 \text { more to } \\
109 \text { more) }\end{array}$ \\
\hline Treatment default & 1,325 (7 RCTs) & $\oplus \oplus \bigcirc \bigcirc$ LOW a,b & $0.63(0.45-0.89)$ & 126 per 1,000 & $\begin{array}{c}46 \text { fewer per } 1,000 \text { (69 fewer } \\
\text { to } 14 \text { fewer) }\end{array}$ \\
\hline Treatment failure & 1,245 (6 RCTs) & $\oplus \oplus \bigcirc \bigcirc \operatorname{LOW} a, b$ & $0.78(0.44-1.40)$ & 50 per 1,000 & $\begin{array}{c}11 \text { fewer per } 1,000 \text { ( } 28 \text { fewer } \\
\text { to } 20 \text { more) }\end{array}$ \\
\hline Death & 1,238 (6 RCTs) & $\oplus \oplus \bigcirc \bigcirc$ LOW a,b & $0.98(0.61-1.57)$ & 68 per 1,000 & $\begin{array}{c}1 \text { fewer per } 1,000 \text { ( } 27 \text { fewer to } \\
39 \text { more) }\end{array}$ \\
\hline
\end{tabular}

95\%Cl: 95\% confidence interval; RCTs: randomized controlled trials; RR: risk ratio.

Note: a. most of information was provided by studies with some limitations (no information about allocation concealment, blinding, and with biases from other sources); b. differences in study populations and interventions might influence directness of evidences; c. high unexplained heterogeneity observation.

\section{Figure 3}

Forest plots for comparison of social protection interventions versus tuberculosis usual care.

3a) Outcome: treatment success

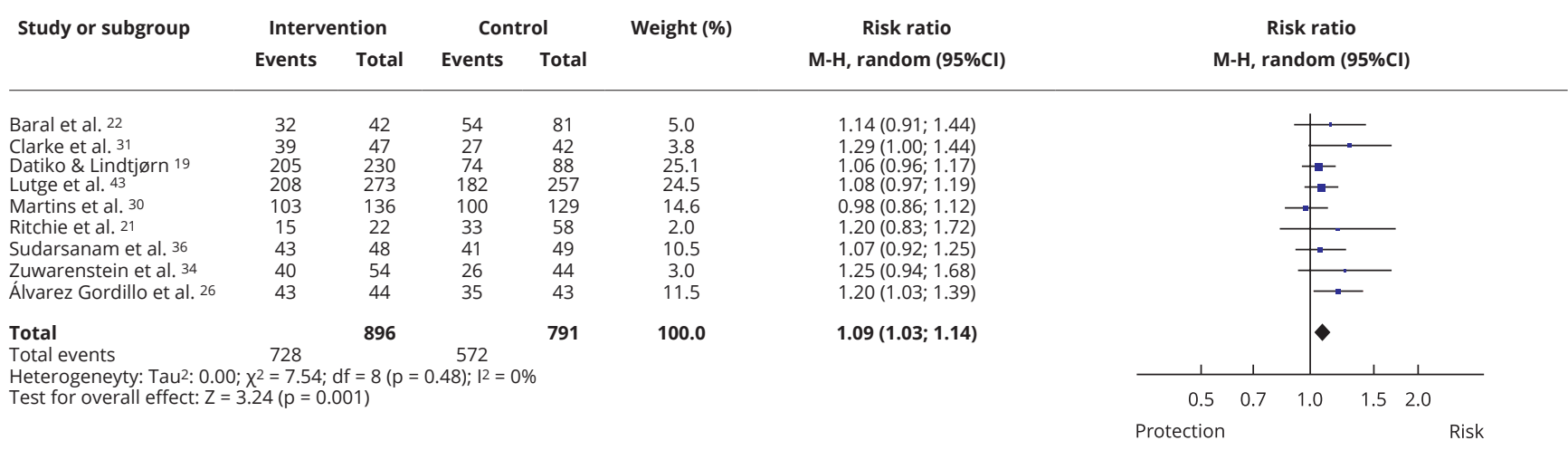

(continues) 
Figure 3 (continued)

3b) Outcome: cure

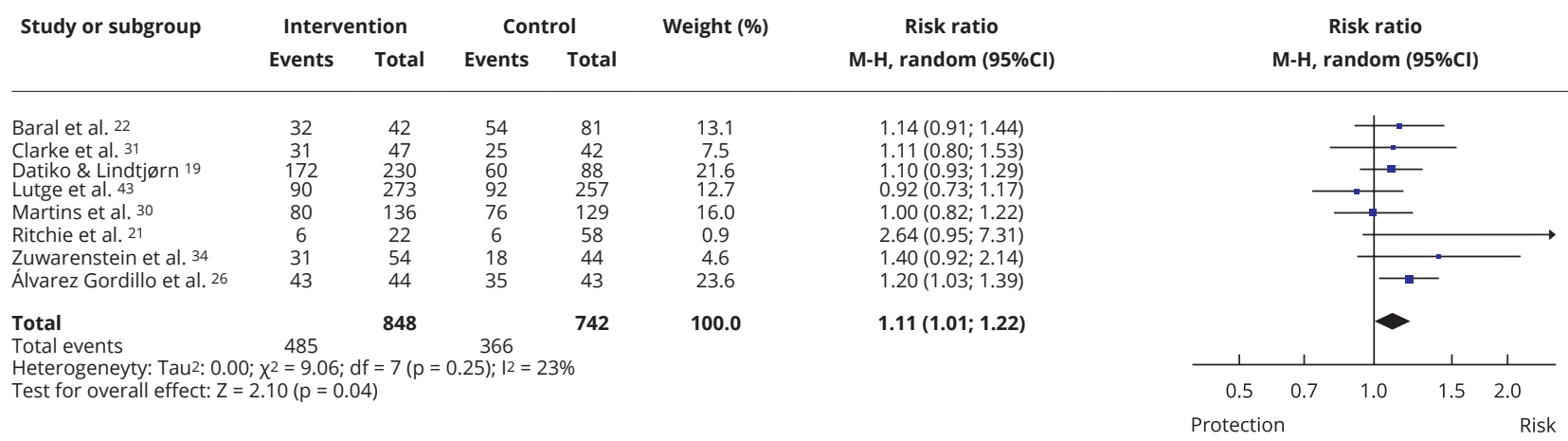

3c) Outcome: default

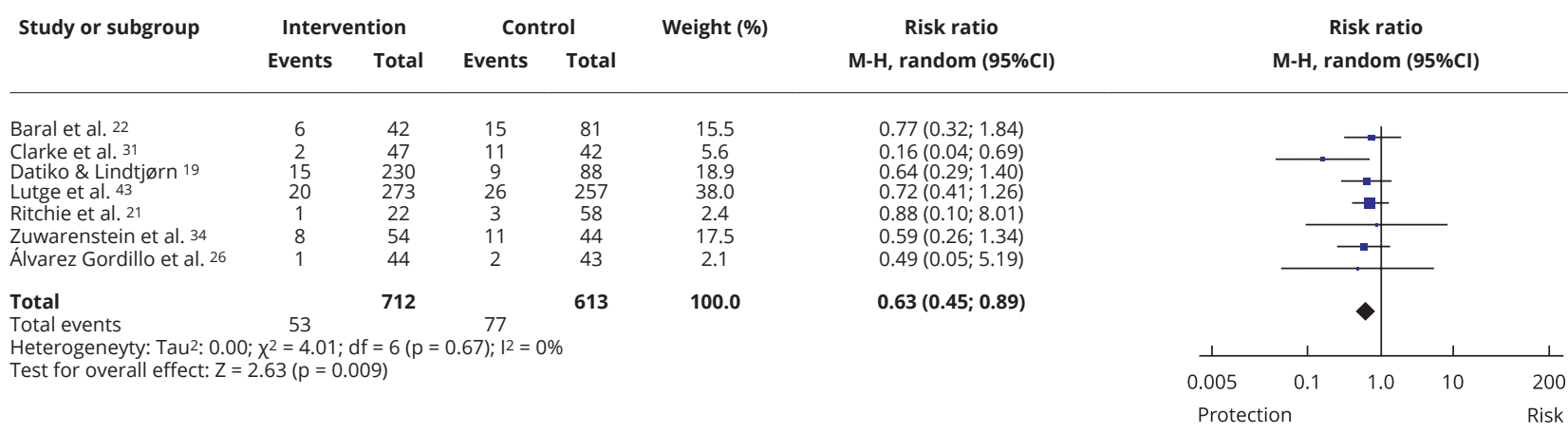

3d) Outcome: failure

\begin{tabular}{|c|c|c|c|c|c|c|}
\hline \multirow[t]{2}{*}{ Study or subgroup } & \multicolumn{2}{|c|}{ Intervention } & \multicolumn{2}{|c|}{ Control } & \multirow[t]{2}{*}{ Weight (\%) } & \multirow{2}{*}{$\begin{array}{c}\text { Risk ratio } \\
\mathrm{M}-\mathrm{H} \text {, random }(95 \% \mathrm{Cl})\end{array}$} \\
\hline & Events & Total & Events & Total & & \\
\hline Baral et al. 22 & 2 & 42 & 4 & 81 & 12.4 & $0.96(0.18 ; 5.05)$ \\
\hline Clarke et al. 31 & 4 & 47 & 2 & 42 & 12.6 & $1.79(0.34 ; 9.26)$ \\
\hline Datiko \& Lindtjørn 19 & 2 & 230 & 0 & 88 & 3.7 & $1.93(0.09 ; 39.73)$ \\
\hline Lutge et al. 43 & 10 & 273 & 15 & 257 & 55.9 & $0.63(0.29 ; 1.37)$ \\
\hline Zuwarenstein et al. 34 & 3 & 54 & 2 & 44 & 11.2 & $1.22(0.21 ; 6.99)$ \\
\hline Álvarez Gordillo et al. 26 & 0 & 44 & 5 & 43 & 4.2 & $0.09(0.01 ; 1.56)$ \\
\hline Total & & 690 & & 555 & 100.0 & $0.78(0.44 ; 1.40)$ \\
\hline $\begin{array}{l}\text { Total events } \\
\text { Heterogeneyty: Tau2: } 0.0 \\
\text { Test for overall effect: } Z\end{array}$ & $\begin{array}{c}21 \\
2=4.22 \\
32(p=c\end{array}$ & $=5$ & $\begin{array}{c}28 \\
.52) ; 12=\end{array}$ & & & \\
\hline
\end{tabular}

Risk ratio $\mathrm{M}-\mathrm{H}$, random $(95 \% \mathrm{Cl})$

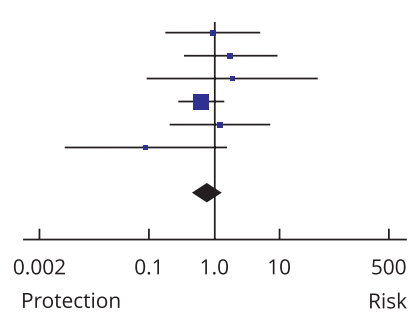

(continues) 
3e) Outcome: death

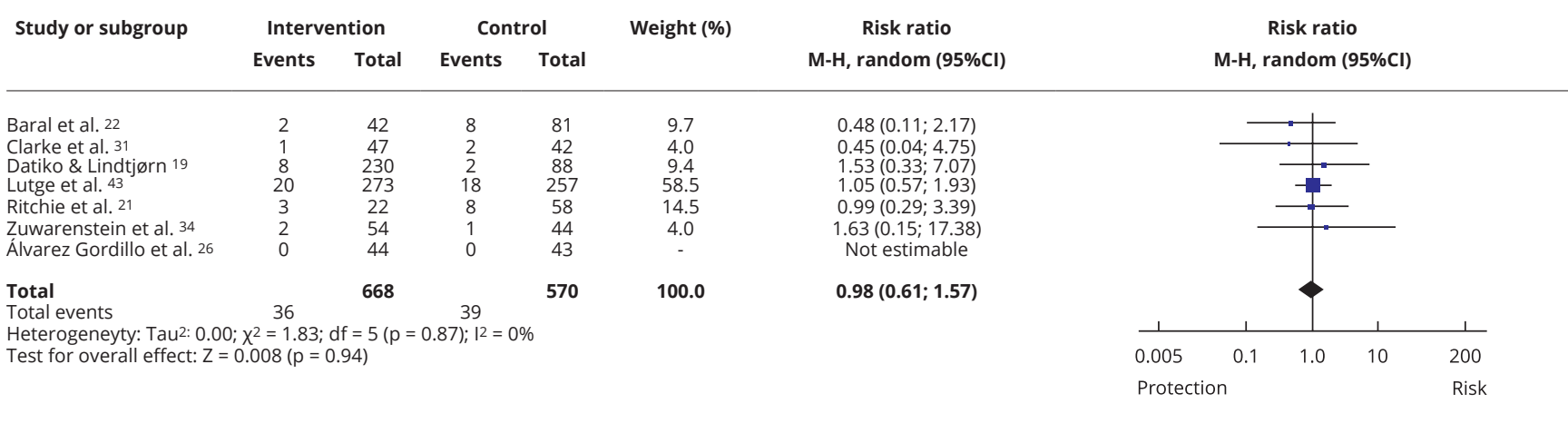

95\%Cl: 95\% confidence interval; M-H: Mantel-Haenszel method.

Despite treatment success being considered a primary outcome, SPS showed a higher effect size against treatment default. This finding can be explained by the capacity that social protection has to increase health care access, leading to better treatment adherence through mechanisms to cope with financial hardship due to TB, alleviate poverty and reduce social vulnerability 1,44.

Because of the disease, individuals and families with TB face direct and indirect costs that can be reduced by SPS. Direct costs include expenses with transport to and from the health facilities, medication, exams or consultations incurred by individuals. Indirect costs are associated with income losses that can account for almost $50 \%$ of total family expenses (e.g. illness-related work absences) 44,45 .

Social protection covering TB patients can provide means for these individuals to compensate catastrophic expenditures and reduce treatment default, especially for the poorest. They can be implemented in different ways, such as material incentives, cash transfers or food security programs 1 . Evidences from RCTs conducted in the United States show that incentives can contribute to patient attendance in TB health care facilities, improving treatment adherence 46.

We noticed that SPS are not limited to cash transfers. In this meta-analysis, financial and non-financial interventions also were effective in protecting against default, leading to treatment success. The scope of social protection includes economic support, food security and nutrition 23,27,28,30,36,37, psychological support and health education approaches 22,26, social mobilization 35 and training of volunteers to act as patient's supporters 19,28,31,32,33,34.

Despite these relevant results, there were some limitations to our meta-analyses. First, the limited number of available studies on social protection effects on TB treatment outcomes. Second, possible uncontrolled biases in the individual studies. Third, methodological limitations of reviewed studies and diversity of their interventions also might have affected our results. Future research based on well-designed randomized studies covering low and middle-income populations might provide better quality evidences.

In order to reduce study selection bias in our meta-analyses, we followed the eligibility criteria previously established in the review protocol. The main differences between this review and the protocol were: inclusion of observational studies in qualitative synthesis and an updated list of countries with high burden of TB. The first change aimed to strength our body of evidences about SPS and TB treatment outcomes. The second change was made to meet WHO current recommendations. In addition, we used Downs \& Black 15 instrument to perform a quality assessment of all reviewed studies, since it can be applied not only to RCTs. Finally, we did not present all planned subgroup analyses, since heterogeneity did not substantially affect our results. 
Concerning publication bias, we agree that funnel plots (available in the supplemental material - cf. Appendix 2: https://www.4shared.com/web/preview/pdf/BVpsBkhoca?) do not ensure its absence but suggest its presence. Furthermore, we could not find unpublished studies and relevant materials in grey literature, strengthening the argument of absence of publication bias in the analyzed studies.

\section{Conclusions}

Our findings endorse the premises that social protection can contribute to TB treatment success, especially improving its adherence. The reach of goals for TB elimination after 2015 depends on the strengthening of social protection among the National Tuberculosis Programs (NTPs) priorities as a main action.

Evidences from our review suggest that low and middle-income countries should extend health coverage to reach the poorest individuals, associated with effective social support, through income transfer mechanisms and comprehensive interventions that may have beneficial impacts on TB outcomes.

Because of the broad scope of social protection, no conclusions can be drawn on the effect of specific interventions. We highlight the uncertainty in the effect estimates provided by the reviewed studies, given the overall low quality of the data. Further well-conducted randomized studies targeting low- and middle-income populations are needed.

\section{Contributors}

K. V. F. Andrade designed the study, searched on databases, selected the articles, performed the statistical analyses and wrote the manuscript. J. S. Nery contributed to quality assessment evidence and to the review of the manuscript. R. A. Souza searched on databases, selected the articles and reviewed the article. S. M. Pereira contributed to all stages of this study, helped in the interpretation of the study findings and reviewed the article.

\section{Acknowledgments}

The authors are grateful for the contributions of the professors Mauricio Lima Barreto, from Federal University of Bahia (UFBA)/Oswaldo Cruz Foundation (Fiocruz) and Maria Lucia Fernandes Penna, from Rio de Janeiro State University (UERJ). We also thank Joney Ribeiro Gonçalves for the grammatical review of this manuscript. This study was supported by Brazilian National Research Council (CNPq) process n. 404030/2012-2. 


\section{References}

1. Lönnroth K, Glaziou P, Weil D, Floyd K, Uplekar M, Raviglione M. Beyond UHC: monitoring health and social protection coverage in the context of tuberculosis care and prevention. PLoS Med 2014; 11:e1001693.

2. Word Health Organization. Global tuberculosis report 2016. Geneva: Word Health Organization; 2016.

3. Word Health Organization. Global strategy and targets for tuberculosis prevention care and control after 2015. Geneva: Word Health Organization; 2015.

4. Reeves A, Basu S, McKee M, Stuckler D, Sandgren A, Semenza J. Social protection and tuberculosis control in 21 European countries, 1995-2012: a cross-national statistical modelling analysis. Lancet Infect Dis 2014; 14:1105-12.

5. Secretaria de Vigilância em Saúde, Ministério da Saúde. Perspectivas brasileiras para o fim da tuberculose como problema de saúde pública. Boletim Epidemiológico 2016; 47(13).

6. Benatar SR, Upshur R. Tuberculosis and poverty: what could (and should) be done? Int J Tuberc Lung Dis 2010; 14:1215-21.

7. Chatham House. Social protection interventions for tuberculosis control: the impact, the challenges, and the way forward. London: Chatham House; 2012.

8. Hargreaves JR, Boccia D, Evans CA, Adato M, Petticrew M, Porter J. The social determinants of tuberculosis: from evidence to action. Am J Public Health 2011; 101:654-62.

9. International Labour Organization; United Nations Children's Fund; United Nations Economic Commission for Africa; United Nations Department of Economic and Social Affairs; United Nations Conference on Trade and Development. UN System Task Team on the post-2015 UN development agenda. Social protection: a development priority in the post-2015 UN development agenda. Geneva: International Labour Organization; 2012.

10. Adato M, Hoddinott J. Social protection: opportunities for Africa. Washington DC: International Food Policy Research Institute; 2008. (IFPRI Policy Brief, 5).

11. Boccia D, Hargreaves J, Lönnroth K, Jaramillo E, Weiss J, Uplekar M, et al. Cash transfer and microfinance interventions for tuberculosis control: review of the impact evidence and policy implications. Int J Tuberc Lung Dis 2011; 15 Suppl 2:S37-49.

12. Moher D, Liberati A, Tetzlaff J, Altman DG; The PRISMA Group. Preferred reporting items for systematic reviews and meta-analyses: the PRISMA statement. PLoS Med 2009; 6:e1000097.

13. World Bank. World Bank country and lending groups. http://datahelpdesk.worldbank.org/ knowledgebase/articles/906519-world-bankcountry-and-lending-groups (accessed on 10/ Dec/2015).
14. World Health Organization. Definitions and reporting framework for tuberculosis - 2013 revision. Geneva: World Health Organization; 2013.

15. Downs SH, Black N. The feasibility of creating a checklist for the assessment of the methodological quality both of randomised and nonrandomised studies of health care interventions. J Epidemiol Community Health 1998; 52:377-84.

16. Higgins JPT, Green S, editors. Cochrane handbook for systematic reviews of interventions. Version 5.1.0. London: Cochrane Collaboration; 2011.

17. Schünemann H, Brozek J, Guyatt G, Oxman A. GRADE handbook for grading quality of evidence and strength of recommendations using the GRADE approach. Hamilton: GRADE Working Group; 2013.

18. GRADEpro GDT. GRADEpro guideline development tool. https://gradepro.org (accessed on 10/Dec/2015).

19. Datiko DG, Lindtjorn B. Health extension workers improve tuberculosis case detection and treatment success in Southern Ethiopia: a community randomized trial. PLoS One 2009; 4:e5443.

20. Demissie M, Getahun H, Lindtjørn B. Community tuberculosis care through "TB clubs" in rural North Ethiopia. Soc Sci Med 2003: 56:2009-18

21. Ritchie LMP, Schull MJ, Martiniuk ALC, Barnsley J, Arenovich T, Lettow MV, et al. A knowledge translation intervention to improve tuberculosis care and outcomes in Malawi: a pragmatic cluster randomized controlled trial. Implement Sci 2015; 10:38.

22. Baral SC, Aryal Y, Bhattrai R, King R, Newell JN. The importance of providing counselling and financial support to patients receiving treatment for multi-drug resistant TB: mixed method qualitative and pilot intervention studies. BMC Public Health 2014; 14:46.

23. Gärden BB, Samarina AA, Stavchanskaya I, Alsterlund RR, Övregaard AA, Taganova OO, et al. Food incentives improve adherence to tuberculosis drug treatment among homeless patients in Russia. Scand J Caring Sci 2012; 27:117-22.

24. Jakubowiak WM, Bogorodskaya EM, Borisov SE, Danilova ID, Lomakina OB, Kourbatova EV. Social support and incentives programme for patients with tuberculosis: experience from the Russian Federation. Int J Tuberc Lung Dis 2007; 11:1210-5.

25. Sripad A, Castedo J, Danford N, Zaha R, Freile C. Effects of Ecuador's national monetary incentive program on adherence to treatment for drug-resistant tuberculosis. Int J Tuberc Lung Dis 2014; 18:44-8. 
26. Álvarez Gordillo GC, Álvarez Gordillo JF, Dorantes Jiménez JE. Estrategia educativa para incrementar el cumplimiento del régimen antituberculoso en Chiapas, México. Rev Panam Salud Pública 2003; 14:402-8.

27. Ciobanu A, Domente L, Soltan V, Bivol S, Severin L, Plesca V, et al. Do incentives improve tuberculosis treatment outcomes in the Republic of Moldova? Public Health Action 2014; 4 Suppl 2:S59-63.

28. Rocha C, Montoya R, Zevallos K, Curatola A, Ynga W, Franco J, et al. The Innovative Socioeconomic Interventions Against Tuberculosis (ISIAT) project: an operational assessment. Int J Tuberc Lung Dis 2011; 15 Suppl 2:S50-7.

29. Kliner M, Canaan M, Ndwandwe SZ, Busulwa F, Welfare W, Richardson M, et al. Effects of financial incentives for treatment supporters on tuberculosis treatment outcomes in Swaziland: a pragmatic interventional study. Infect Dis Poverty 2015; 4:29.

30. Martins N, Morris P, Kelly PM. Food incentives to improve completion of tuberculosis treatment: randomised controlled trial in Dili, Timor-Leste. BMJ 2009; 339:b4248.

31. Clarke M, Dick J, Zwarenstein M, Lombard CJ, Diwan VK. Lay health worker intervention with choice of DOT superior to standard TB care for farm dwellers in South Africa: a cluster randomised control trial. Int J Tuberc Lung Dis 2005; 9:673-9.

32. Singh AA, Parasher D, Shekhavat GS, Sahu S, Wares DF, Granich R. Effectiveness of urban community volunteers in directly observed treatment of tuberculosis patients: a field report from Haryana, North India. Int J Tuberc Lung Dis 2004; 8:800-2.

33. Soares ECC, Vollmer WM, Cavalcante SC, Pacheco AG, Saraceni V, Silva JS, et al. Tuberculosis control in a socially vulnerable area: a community intervention beyond DOT in a Brazilian favela. Int J Tuberc Lung Dis 2013; 17:1581-6.

34. Zwarenstein M, Schoeman JH, Vundule C, Lombard CJ, Tatley M. A randomised controlled trial of lay health workers as direct observers for treatment of tuberculosis. Int J Tuberc Lung Dis 2000; 4:550-4.

35. Ngamvithayapong-Yanai J, Luangjina S, Nedsuwan S, Kantipong P, Wongyai J, Ishikawa N. Engaging women volunteers of high socioeconomic status in supporting socioeconomically disadvantaged tuberculosis patients in Chiang Rai, Thailand. Western Pac Surveill Response J 2013; 4:34-8.
36. Sudarsanam TD, John J, Kang G, Mahendri V, Gerrior J, Franciosa M, et al. Pilot randomized trial of nutritional supplementation in patients with tuberculosis and HIV-tuberculosis coinfection receiving directly observed shortcourse chemotherapy for tuberculosis. Trop Med Int Health 2011; 16:699-706.

37. Cantalice Filho JP. Efeito do incentivo alimentício sobre o desfecho do tratamento de pacientes com tuberculose em uma unidade primária de saúde no município de Duque de Caxias, Rio de Janeiro. J Bras Pneumol 2009; 35:992-7.

38. Lönnroth K, Aung T, Maung W, Kluge H, Uplekar M. Social franchising of TB care through private GPs in Myanmar: an assessment of treatment results, access, equity and financial. Health Policy Plan 2007; 22:156-66.

39. Wei X, Zou G, Yin J, Walley J, Yang H, Kliner $\mathrm{M}$, et al. Providing financial incentives to rural-to-urban tuberculosis migrants in Shanghai: an intervention study. Infect Dis Poverty 2012; 1:9.

40. Yao H, Wei X, Liu J, Zhao J, Hu D, Walley JD. Evaluating the effects of providing financial incentives to tuberculosis patients and health providers in China. Int $\mathrm{J}$ Tuberc Lung Dis 2008; 12:1166-72.

41. Zou G, Wei X, Witter S, Yin J, Walley J, Liu S, et al. Incremental cost-effectiveness of improving treatment results among migrant tuberculosis patients in Shanghai. Int J Tuberc Lung Dis 2013; 17:1056-64.

42. Torrens AW, Rasella D, Boccia D, Maciel ELN, Nery JS, Olson ZD, et al. Effectiveness of a conditional cash transfer programme on TB cure rate: a retrospective cohort study in Brazil. Trans R Soc Trop Med Hyg 2016; 110:199206.

43. Lutge E, Lewin S, Volmink J, Friedman I, Lombard C. Economic support to improve tuberculosis treatment outcomes in South Africa: a pragmatic cluster-randomized controlled trial. Trials 2013; 14:154.

44. Foster N, Vassall A, Cleary S, Cunnama L, Churchyard G, Sinanovic E. The economic burden of TB diagnosis and treatment in South Africa. Soc Sci Med 2015; 130C:42-50.

45. Tanimura T, Jaramillo E, Weil D, Raviglione M, Lonnroth K. Financial burden for tuberculosis patients in low- and middle-income countries: a systematic review. Eur Respir J 2014; 43:1763-75.

46. Lutge EE, Wiysonge CS, Knight SE, Sinclair D, Volmink J. Incentives and enablers to improve adherence in tuberculosis. Cochrane Database Syst Rev 2015; 9:CD007952. 


\section{Resumo}

A tuberculose (TB) é uma doença infecciosa associada à pobreza que afeta milhões de pessoas no mundo. As evidências sugerem que estratégias de proteção social podem melhorar os desfechos do tratamento da TB. O estudo teve como objetivo resumir essas evidências através de uma revisão sistemática da literatura e uma meta-análise. Foram buscados estudos realizados em países de renda baixa e média ou com carga alta de TB, publicados entre 1995 e 2016. A revisão foi realizada através de uma busca em PubMed/MEDLINE, Scopus, Web of Science, ScienceDirect e LILACS. Incluimos apenas os estudos que investigavam os efeitos das estratégias de proteção social sobre os desfechos do tratamento da TB. Foram incluídos 25 estudos na síntese qualitativa. As meta-análises foram realizadas com 9 estudos randomizados e controlados, totalizando 1.687 participantes. Os resultados mostraram que as estratégias de proteção social estavam associadas ao sucesso do tratamento da TB $(R R=1,09 ;$ IC95\%: 1,03-1,14), à cura dos pacientes de TB (RR = 1,11; IC95\%: 1,01-1,22) e à redução do risco de abandono do tratamento $(R R=0,63$; IC95\%: 0,45-0,89). Não detectamos os efeitos das estratégias de proteção social sobre a falha terapêutica ou mortalidade. Os achados mostram que as estratégias de proteção social podem melhorar os desfechos do tratamento em países com renda baixa e média ou com alta carga da doença. Entretanto, a qualidade das evidências com relação a essas estimativas de efeito é baixa, e são necessários mais estudos randomizados e bem conduzidos.

Tuberculose; Resultado do Tratamento; Seguridade Social; Política Pública

\section{Resumen}

La tuberculosis (TB) es una enfermedad infecciosa, característica de la pobreza, que afecta a millones de personas en todo el mundo. Las evidencias sugieren que las estrategias de protección social (EPS) pueden mejorar los resultados del tratamiento de la TB. El objetivo de este estudio ha sido resumir tales evidencias, a través de una revisión sistemática de la literatura y metaanálisis. Buscamos estudios realizados en países de baja renta o ingresos medios y con altas tasas de morbilidad por TB, publicados durante 1995-2016. La revisión la llevamos a cabo realizando búsquedas en PubMed/ MEDLINE, Scopus, Web of Science, ScienceDirect y LILACS. Incluimos sólo estudios que investigaron los efectos de las EPS en los resultados de los tratamientos contra la TB. Seleccionamos 25 estudios para realizar su sintesis cualitativa. Realizamos metaanálisis con 9 ensayos controlados aleatorios, incluyendo a un total de 1.687 participantes. Los resultados agrupados mostraron que las EPS estaban asociadas con tratamientos exitosos contra la $T B(R R=1,09$; 95\%CI: 1,03-1,14), la curación en pacientes de $T B(R R=1,11 ; 95 \% C I$ : 1,01-1,22) y con la reducción en el riesgo de abandono del tratamiento de $T B(R R=0,63 ; 95 \% C I$ : 0,45-0,89). No detectamos efectos de las EPS en los resultados de fracaso del tratamiento y muerte por TB. Estos hallazgos revelaron que las EPS podrían mejorar los resultados de los tratamientos por TB en las economias de países con baja renta o ingresos medios, o países con altas tasas de esta enfermedad. No obstante, la calidad general de las evidencias, en relación con estos resultados, es baja e indica que son necesarios más estudios controlados aleatorios bien realizados.

Tuberculosis; Resultado del Tratamiento; Bienestar Social; Politica Pública
Submitted on 31/Aug/2016

Final version resubmitted on 06/Oct/2017 Approved on 09/Oct/2017 